\title{
Predictors of sleep disturbance in heroin users receiving methadone maintenance therapy: a naturalistic study in Taiwan
}

This article was published in the following Dove Press journal:

Neuropsychiatric Disease and Treatment

\author{
Dian-Jeng $\mathrm{Li}^{1,2}$ \\ Kuan-Shang Chung' \\ Hung-Chi Wu' \\ Chih-Yao Hsu' \\ Cheng-Fang Yen ${ }^{2,3}$ \\ 'Department of Addiction Science, \\ Kaohsiung Municipal Kai-Syuan \\ Psychiatric Hospital, Kaohsiung, \\ Taiwan; ${ }^{2}$ Graduate Institute of \\ Medicine, College of Medicine, \\ Kaohsiung Medical University, \\ Kaohsiung, Taiwan; ${ }^{3}$ Department \\ of Psychiatry, Kaohsiung Medical \\ University Hospital, Kaohsiung, Taiwan
}

Correspondence: Cheng-Fang Yen Department of Psychiatry, Kaohsiung Medical University Hospital, 100 Tzyou Ist Road, Kaohsiung 807, Taiwan Tel +886 7 3 I 2 । I $0 \mid$ ext 6822 Fax +88673I3 476I

Email chfaye@cc.kmu.edu.tw

\begin{abstract}
Introduction: Sleep disturbance is a major health concern for heroin users receiving methadone maintenance treatment (MMT). The present study was aimed to investigate the predictors for new-onset clinically predominant sleep disturbance (CPSD) among heroin users receiving MMT.

Methods: This 2-year retrospective study included 152 individuals (127 males and 25 females) with heroin use disorder who visited our MMT clinics for the first time. A univariate Cox proportional hazards regression model (Cox model) was used to estimate the potential factors of subsequent CPSD, followed by a multivariate Cox model to identify significant predictors of CPSD after adjusting for other covariates.

Results: Twenty-nine (19.1\%) participants developed CPSD during the 2-year period. After forward selection in the Cox model, earlier age at onset of heroin exposure $(\mathrm{OR}=0.95 ; P=0.044)$, lower attendance rate $(\mathrm{OR}=0.04 ; P=0.03)$, greater maximum dose of methadone $(\mathrm{OR}=1.01$; $P=0.022)$, and shorter time to maximum methadone dose $(\mathrm{OR}=0.98 ; P=0.007)$ were significantly associated with new-onset CPSD.
\end{abstract}

Conclusion: We identified predictors that were significantly associated with new-onset CPSD, and clinicians should be aware of sleep disturbance in heroin users receiving MMT with these risk factors. Future studies are necessary to verify our findings and extend the applicability.

Keywords: MMT, new-onset sleep disturbance, heroin, CPSD

\section{Introduction}

Sleep disturbance is a major health problem affecting more than one-third of the general population, ${ }^{1-3}$ and it has been reported to influence the quality of life, functions of life, health, and mortality. ${ }^{4-6}$ Sleep disturbance has also been associated with physical illnesses, ${ }^{7}$ cognitive difficulties, the risk of injury, and motor vehicle accidents. ${ }^{8}$ The etiology of sleep disturbance is complicated and multidimensional. Patients suffering from sleep problems are associated with comorbidities such as psychiatric disorders, including depression. ${ }^{9}$ It has been estimated that $10 \%-15 \%$ of patients with chronic sleep disturbance also have comorbid substance abuse. ${ }^{10}$ The relationship between substance use and sleep disturbance may be bidirectional, in that substance use may induce sleep problems, and sleep disturbance may increase the risk of relapse of substance use. ${ }^{11}$

Heroin dependence is a serious public health concern affecting many people worldwide. According to the World Drug Report, opioids are the most harmful drug type, accounting for $70 \%$ of the negative health impact associated with drug use disorders worldwide. ${ }^{12}$ Heroin dependence can result in a heavy socioeconomic burden, 
including the need for medical care, lost productivity, and cost of social welfare. ${ }^{13}$ Previous studies have demonstrated that exposure to heroin can lead to shortening of sleep latency, reduction in total sleep time and sleep efficiency, and inhibition of rapid eye movement (REM) and non-REM phases of sleep. ${ }^{14-16}$ Sleep disturbance affects both heroin abusers and heroin users receiving methadone maintenance therapy (MMT). Methadone, a synthetic opioid, has been approved as an effective therapeutic strategy for opioid users due to its interaction with opioid receptors. ${ }^{17}$ Research has shown that perceived sleep problems exist in up to $75 \%$ of heroin users receiving MMT. ${ }^{18}$ One prospective study reported that patients maintained on methadone or buprenorphine had high levels of sleep impairment, including not getting the amount of sleep they needed, not sleeping enough to feel rested, trouble falling asleep, and falling back asleep after waking. ${ }^{19}$

Several mechanisms have been postulated to explain insomnia among heroin users receiving MMT, and heroin users receiving MMT have been shown to suffer from more sleep architecture abnormalities and central sleep apnea than controls. $^{20,21}$ In addition, Nguyen et al reported that patients receiving MMT had higher respiratory variability during sleep than healthy controls, indicating sleep-disordered breathing. ${ }^{22}$ Moreover, the administration of opioids can decrease levels of adenosine, a neurochemical modulator of the homeostatic drive for sleep, which is dependent on the opioid receptor $\mu-1$ (OPRM1) gene, subsequently resulting in sleep disturbance. ${ }^{23}$ The possibility that lower levels of adenosine may be associated with sleep disturbance in heroin users receiving MMT is further supported by the observation that heroin users receiving MMT fail to show typical recovery responses after a sleep-deprivation challenge. ${ }^{24}$ From a genetic point of view, Wang et al reported that some single nucleotide polymorphisms in OPRM1 may be associated with the sleep disturbance found in patients receiving MMT. ${ }^{25}$ Furthermore, heroin decreases acetylcholine release in some brain regions such as the pontine reticular formation, thereby further inhibiting REM sleep. ${ }^{26}$ Heroin can also suppress inhibitory GABAergic transmission in the dorsal raphe nucleus, promoting wakefulness. ${ }^{27}$ The association between sleep disturbance and MMT may be mediated by depression, which is prevalent in both groups. ${ }^{28}$ Withdrawal from heroin along with methadone detoxification may also contribute to sleep disturbance. ${ }^{29}$ It is important to assess sleep disturbance in heroin users receiving MMT because it may adversely affect treatment outcomes, mental health, quality of life, ${ }^{30-32}$ and adherence to treatment. ${ }^{33}$
Although previous studies have discussed sleep disturbance in heroin users receiving MMT, few studies have investigated new-onset sleep disturbance in heroin users without sleep disturbance at the initiation of MMT. Furthermore, most of these studies have investigated self-reported sleep disturbance but not clinically evaluated sleep disturbance. ${ }^{18,34}$ It would be beneficial for psychiatrists to be able to evaluate the factors associated with new-onset sleep disturbance. A few studies have investigated the factors associated with sleep disturbance; ${ }^{18,35}$ however, studies investigating the early predictors of sleep disturbance in patients receiving MMT are lacking. Thus, this retrospective, 2-year follow-up study was aimed to investigate the predictors of new-onset sleep disturbance based on clinicians' evaluations of heroin users receiving MMT in Taiwan. In comparison with previous studies using self-reported questionnaires, ${ }^{18,34}$ we investigated clinically predominant sleep disturbance (CPSD), indicating sleep disturbance requiring clinical intervention. The current study provides another point of view to depict sleep disturbance. We hypothesized that demographic characteristics and characteristics of heroin use and MMT may predict new-onset sleep disturbance, and that clinicians could identify these factors in clinical practice.

\section{Methods}

\section{Ethics}

This study was approved by the Institutional Review Board of Kai-Syuan Psychiatric Hospital (KSPH-2017-07) according to the current revision of the Declaration of Helsinki and national legal requirements (Human Subjects Research Act, Taiwan). We did not need formal informed consent as deidentified data were used in the analysis.

\section{Participants}

This 2-year retrospective study included individuals with heroin dependence who visited the MMT clinics of Kai-Syuan Psychiatric Hospital for the first time for MMT from January 2015 to August 2017. This hospital is the core psychiatric hospital in southern Taiwan and provides multidimensional psychiatric treatment and services, including admissions, outpatient services, emergency services, and community services. The hospital also offers multiple specialist services, including treatment for addiction. The inclusion criteria were patients who 1) were at least 18 years of age; 2) met the DSM-IV-TR or DSM-5 criteria for heroin use disorder as assessed by psychiatrists; and 3) had positive findings of opioids in a urine test before treatment. We excluded patients with a history of schizophrenia, neurocognitive disorders, neurodevelopmental disorders, major 
depressive disorder, bipolar disorder, and mental disorders due to medical conditions according to the DSM-IV-TR or DSM-5. Patients diagnosed with these disorders were excluded as we aimed to examine the association between sleep problems and MMT, and these disorders may have been comorbid with sleep disturbance. Furthermore, patients were excluded if they had ever taken sleeping pills or been diagnosed with sleep disorders before starting MMT. Patients with substance use were also excluded except for those with opioid and nicotine use.

All assessments were made by psychiatrists at our hospital. All of the patients underwent diagnostic interviews to evaluate psychiatric comorbidities according to the DSMIV-TR or DSM-5, along with a urine test for illegal substances. A physical examination, hematological test, electrocardiography, and chest X-ray were performed to exclude undesirable medical conditions before entering MMT. Additional urine tests for other substances were performed according to the clinical judgment of the psychiatrists during MMT. We prescribed methadone according to official practice guidelines for heroin use disorder. The patients were prescribed with methadone at an initial daily dose of $10-40 \mathrm{mg}$ according to the severity of withdrawal symptoms and medical conditions. The prescribed dose was then slowly titrated to a maximal maintenance dose. After relieving the withdrawal symptoms, the patients were encouraged to gradually taper the daily dose of MMT. The difference in titration or tapering dose did not exceed $10 \mathrm{mg}$ per day. The patients revisited the MMT clinic once per week in the first few months, then once per month after they had achieved a maximal maintenance dose. If the patients wished to titrate or taper the dose of methadone more than two times in 1 month, they were asked to revisit our clinic once or twice per month for detailed assessments. Patients who were absent for more than 6 days in 1 month or for three continuous days were also asked to revisit our clinic for intensive assessments and adjustments of the dosage by their psychiatrist once per week. Furthermore, those who were absent for 14 continuous days were discontinued from our MMT program.

\section{Outcome measures and predictors}

We retrospectively collected data from the hospital's database of electronic records. The primary outcome was time to new-onset CPSD, defined as acute onset of sleep disturbance requiring pharmacological or nonpharmacological treatment either at initiation or during the retrospective period of MMT. CPSD was assessed by the treating psychiatrists at the MMT clinics. When patients complained about sleep problems, the treating psychiatrists would provide an appropriate intervention. Those who were treated with either psychoeducative psychotherapy or psychotropic medication were defined as patients with CPSD. We also collected several potential predictors and controlled for their effects on new-onset CPSD. These potential predictors included sex (male or female), employment status (employed or unemployed), marital status (married, single, and divorced or widowed), human immunodeficiency virus infection status, age at onset of heroin exposure, age at initiating MMT, education level, maximum MMT dose, time to maximum MMT dose, and attendance rate. The attendance rate was defined as the percentage of days the patients actually attended MMT divided by the days they should have attended MMT, and it was calculated from the initial attending date to the date of new-onset CPSD, being lost to follow-up, or the end of the retrospective period.

\section{Statistical analysis}

We initially conducted descriptive statistics to summarize the clinical characteristics at baseline. For the first step, we used a univariate Cox proportional hazards regression model of survival analysis to investigate potential factors associated with the time to CPSD. Statistically significant factors $(P<0.05)$ obtained from the first step were then entered into a forward multivariate Cox proportional hazards regression model to identify definite risk factors after adjusting for other covariates. In addition, Pearson correlation analysis was used to detect possible collinearity between factors. All tests were two-tailed, and statistical significance was set at $P<0.05$. All data were processed using SPSS version 17.0 for Windows (SPSS Inc., Chicago, IL, USA).

\section{Results \\ Patient variables}

One hundred and seventy-eight heroin users with MMT were initially screened. Among them, 26 were excluded due to comorbidity with schizophrenia $(n=1)$, major depressive disorder $(n=8)$, bipolar disorder $(n=4)$, other substance use $(n=10)$, and mood disorder comorbid with other substance use $(n=3)$. Finally, a total of 152 heroin users receiving MMT were included into our analysis after screening for the inclusion and exclusion criteria. The characteristics are listed in Table 1.

\section{Predictors of CPSD}

Twenty-nine of the 152 participants $(19.1 \%)$ had newonset CPSD. All of them were treated with psychoeducative psychotherapy and psychotropic medications as 
Table I Potential predictors affecting the time to developing CPSD identified by univariate Cox proportional hazards regression analysis $(n=152)$

\begin{tabular}{|c|c|c|c|c|c|c|}
\hline Variable & $\mathbf{n}$ & $\%$ & B & Odds ratio & $95 \% \mathrm{Cl}$ & $P$-value \\
\hline \multicolumn{7}{|l|}{ Sex } \\
\hline Male & 127 & 83.6 & 0.27 & 1.31 & $0.46-3.77$ & 0.613 \\
\hline Female & 25 & 16.4 & & & & \\
\hline \multicolumn{7}{|l|}{ HIV infection } \\
\hline Yes & 9 & 5.9 & 0.25 & 1.28 & $0.30-5.38$ & 0.739 \\
\hline No & 143 & 94.1 & & & & \\
\hline \multicolumn{7}{|l|}{ Nicotine use } \\
\hline User & 124 & 81.6 & 1.94 & 6.94 & $0.94-51.04$ & 0.057 \\
\hline Nonuser & 28 & 18.4 & & & & \\
\hline \multicolumn{7}{|l|}{ Marital status } \\
\hline Married & 95 & 62.5 & - & - & - & - \\
\hline Single & 34 & 22.4 & -0.20 & 0.82 & $0.33-2.03$ & 0.663 \\
\hline Divorced & 23 & 15.1 & -0.52 & 0.60 & $0.18-2.01$ & 0.406 \\
\hline \multicolumn{7}{|l|}{ Employment status } \\
\hline Employed & 145 & 95.4 & 0.39 & 1.47 & $0.20-10.83$ & 0.705 \\
\hline \multirow[t]{2}{*}{ Unemployed } & 7 & 4.6 & & & & \\
\hline & Mean & SD & B & Odds ratio & $95 \% \mathrm{Cl}$ & $P$-value \\
\hline Age at initial MMT (years) & 42.11 & 7.8 & 0.03 & 1.03 & $0.97-1.08$ & 0.378 \\
\hline Age at onset of heroin exposure (years) & 35.6 & 11.4 & -0.10 & 0.91 & $0.87-0.95$ & $<0.001$ \\
\hline Attendance rate (\%) & 72.9 & 15.6 & -4.54 & 0.01 & $0.00 I-0.15$ & 0.001 \\
\hline Maximum of methadone doses (mg/day) & 69.7 & 30.0 & 0.01 & 1.01 & $1.00-1.02$ & 0.006 \\
\hline Time to maximal methadone doses (days) & 83.6 & 124.4 & -0.03 & 0.97 & $0.96-0.99$ & 0.006 \\
\hline Educational level (years) & 10.3 & 2.3 & -0.03 & 0.97 & $0.83-1.14$ & 0.727 \\
\hline
\end{tabular}

Note: Bold values indicate statistical significance, $P<0.05$.

Abbreviations: CPSD, clinically predominant sleep disturbance; MMT, methadone maintenance therapy.

follows: lorazepam $(n=4 ; 13.8 \%)$, flurazepam $(n=6 ; 20.7 \%)$, estazolam $(n=12 ; 41.4 \%)$, brotizolam $(n=2 ; 6.7 \%)$, and zolpidem/zopiclone $(n=5 ; 17.2 \%)$. Using univariate Cox regression analysis in the first step, an earlier age at the onset of heroin exposure $(\mathrm{OR}=0.91 ; P<0.001)$, lower attendance rate (OR $=0.01 ; P=0.001)$, greater maximum dose of methadone (OR $=1.01 ; P=0.006)$, and shorter time to maximum methadone dose $(\mathrm{OR}=0.97 ; P=0.006)$ were significantly associated with new-onset CPSD (Table 1). Further analysis using forward multivariate Cox regression analysis revealed that an earlier age at onset of heroin exposure $(\mathrm{OR}=0.95 ; P=0.044)$, lower attendance rate $(\mathrm{OR}=0.04 ; P=0.03)$, greater maximum dose of methadone $(\mathrm{OR}=1.01 ; P=0.022)$, and shorter time to maximum methadone dose $(\mathrm{OR}=0.98 ; P=0.007)$ were all significantly associated with new-onset CPSD (Table 2). Furthermore, Pearson correlation analysis showed that there was no collinearity between time to maximum methadone dose and maximum dose of methadone ( $r=0.157 ; P=0.053)$, time to maximum methadone dose vs age at onset of heroin exposure ( $r=0.057 ; P=0.485$ ), time to maximum methadone dose vs attendance rate $(r=0.157 ; P=0.054)$, or maximum methadone dose vs attendance rate $(r=0.140 ; P=0.085)$. There were significant but slight correlations between attendance rate vs age at onset of heroin exposure $(r=0.22 ; P=0.006)$ and maximum methadone dose vs age at onset of heroin exposure ( $r=-0.18 ; P=0.027$ ). Among them, the effect sizes of significant correlations were low regarding the cutoff values of Pearson correlation.

\section{Discussion}

In the present study, we identified several predictors for the subsequent development of CPSD among heroin users

Table 2 Powerful factors affecting the time to developing CPSD using forward multivariate Cox proportional hazards regression analysis

\begin{tabular}{l|l|l|l|l}
\hline & $\mathbf{B}$ & Odds ratio & $\mathbf{9 5 \%} \mathbf{C l}$ \\
\hline Maximum methadone dose & 0.01 & 1.01 & $1.00-1.02$ & $\mathbf{P}$-value \\
Age at onset of heroin exposure & -0.05 & 0.95 & $0.91-0.99$ \\
Time to maximal methadone dose & -0.03 & 0.98 & $0.96-0.99$ \\
Attendance rate & -3.26 & 0.04 & $0.002-0.74$ & $\mathbf{0 . 0 0 7}$ \\
\hline
\end{tabular}

Note: Bold values indicate statistical significance, $P<0.05$.

Abbreviation: CPSD, clinically predominant sleep disturbance. 
treated with MMT. Sleep disturbance is associated with many problems in heroin users, and our results may help clinicians to identify the early signs of sleep disturbance and implement treatment plans to prevent worsening of sleep status. Previous studies investigating sleep problems among heroin users have often used self-reported questionnaires such as the Pittsburgh Sleep Quality Index (PSQI) and Epworth Sleepiness Scale. ${ }^{36,37}$ However, heroin users may not seek medical assistance. In order to provide another point of view, we used CPSD as the primary outcome. We defined CPSD as "sleep disturbance requiring medical assistance", which indicated more severe sleep disturbance which may have affected the heroin users' health and daily function. Furthermore, the factors we identified in the present study may be an underrecognized problem among heroin users receiving MMT. ${ }^{35}$ As a result, we suggest that clinicians should routinely evaluate the sleep condition of heroin users when prescribing methadone. We found lower rates of co-occurring other substance use and major psychiatric disorders compared with a previous study. ${ }^{38}$ The patients with multiple substance use or major psychiatric disorders may have more easily dropped out from our MMT program at the outpatient department due to repeated admissions, and they would have been prescribed with buprenorphine during admission. Due to the additional treatment costs in these specific patients, it may be difficult for them to enroll in the MMT program due to the policy of National Health Insurance Bureau in Taiwan.

Our results showed that an earlier age at the onset of heroin exposure predicted the subsequent onset of CPSD. Although a previous study demonstrated no association between the age at onset of heroin use and sleep disturbance, ${ }^{39}$ it has also been reported that patients with long-term opioid use frequently have severe sleep-disordered breathing, which in part is central in origin..$^{40,41}$ Furthermore, a longer duration of MMT has been significantly correlated with obstructive sleep apnea. ${ }^{42}$ Our results further support that a long period of heroin exposure can predict the development of sleep disturbance. On the other hand, the mean age at onset of heroin exposure (35.6 years) was much older than in a previous study in Australia (18 years). ${ }^{43}$ However, previous studies have also reported an older age at onset of heroin use ranging from 25 to 30 years in Taiwan, ${ }^{44,45}$ which is closer to our report. Differences in social culture may contribute to the difference between Taiwan and other countries. We also found that a higher maximum dosage of methadone predicted the development of CPSD. A morphine equivalent daily dose of $>200 \mathrm{mg}$ has been strongly associated with the severity of sleep-disordered breathing. ${ }^{46,47}$ A retrospective cohort study reported similar findings to our results and suggested that a higher dosage of opioids was associated with central sleep apnea and ataxic breathing. ${ }^{41} \mathrm{We}$ also found that a shorter time to a maximum dose of methadone predicted subsequent CPSD. Although this factor has rarely been reported, it may also be explained by the association between sleep disturbance and heroin. Patients with a shorter time to a maximum dose of methadone may have a stronger initial desire for heroin, and they may be at risk of higher doses of exposure to heroin before entering MMT.

In the present study, we found a link between a lower attendance rate and subsequent CPSD. This may be because heroin users with low adherence to MMT are at risk of additional heroin abuse, and thus an increased risk of sleep disturbance. A prospective study investigating treatment outcomes of MMT indicated that poor adherence can substantially increase the risk of drug relapse. ${ }^{48}$ Hence, clinicians should make efforts to improve adherence in order to prevent drug relapse and sleep disturbance. Finally, we found that sex and age at initiating MMT were not significantly associated with new-onset CPSD, which is consistent with previous studies $^{18,35}$ that reported no correlations between sex and age at initiating MMT and subjective sleep disturbance as measured by the PSQI. Moreover, several studies have reported a significant association between smoking and sleep problems, ${ }^{49,50}$ and a prospective study also reported that opioid-dependent patients with severe nicotine dependence had a higher risk of severe sleep disturbance. ${ }^{51}$ However, the present study did not find a significant association between nicotine use and CPSD. Further studies focusing on heroin users receiving MMT are needed to explore the association between nicotine use and sleep disturbance.

A strength of this study is that it is a naturalistic observation reflecting clinical practice in the real world. We also identified several predictors that have seldom been reported before for the onset of sleep disturbance in heroin users receiving MMT. However, there are also several limitations to this study. First, as a retrospective chart review, the possibility of missing data limits the outcomes. For example, the pattern of nicotine use was absent from our electronic records, and there were insufficient data concerning the clinical symptoms or polysomnography for sleep disturbance. Furthermore, certain factors were not explored, including close family member substance abuse history and current psychosocial problems. ${ }^{52}$ These factors should be investigated in future studies on the prediction of the onset of CPSD. Second, the heroin users receiving MMT may not have visited our outpatient department for sleep disturbance during 
the retrospective period and may not have been identified to have CPSD. However, as the core psychiatric hospital in southern Taiwan, our hospital provides a wide range of treatments including treatment for addiction and sleep disturbance. Patients with sleep problem who failed to ask for help at our MMT clinics may also limit the interpretation of our study. Third, although patients with substance use except for opioid and nicotine use were initially excluded from the study and from the follow-up period, we only routinely (not less than once per month) performed urine tests for heroin. Psychiatrists made clinical judgment on the participants' use of benzodiazepine or illegal substances other than heroin. Therefore, it is possible that some participants with other substance use were not excluded during the follow-up period. Moreover, undetected exposure to other substances may have confounded the impact of related factors, such as attendance rates. Fourth, the relatively small sample size may have limited the interpretation of the current study. Moreover, the relatively few female patients may have undermined the reliability of gender comparisons. Fifth, there was a lack of early interventions or psychosocial programs in our study. ${ }^{53,54}$ Specific interventions for patients with a younger age at heroin exposure and well-structured sleep hygiene programs should be investigated in future research to elucidate their clinical implications. Finally, as a single-center study, the generalizability of the results may be limited.

\section{Conclusion}

We found that a younger age at heroin exposure, a lower attendance rate of MMT, a higher maximum dosage of methadone, and a shorter time to maximum methadone dose predicted new-onset CPSD. We investigated sleep disturbance requiring clinical assistance, which is different from self-reported sleep disturbance commonly reported in recent publications. Future studies with a larger sample size, longer retrospective period, unmeasured psychological factors, and specific interventions for sleep disturbance are necessary to verify and extend the generalizability of our findings.

\section{Disclosure}

The authors report no conflicts of interest in this work.

\section{References}

1. Ancoli-Israel S, Roth T. Characteristics of insomnia in the United States: results of the 1991 National Sleep Foundation Survey. I. Sleep. 1999; 22(2):S347-S353.

2. Calem M, Bisla J, Begum A, et al. Increased prevalence of insomnia and changes in hypnotics use in England over 15 years: analysis of the 1993, 2000, and 2007 National Psychiatric Morbidity Surveys. Sleep. 2012;35(3):377-384.
3. Walsh JK, Coulouvrat C, Hajak G, et al. Nighttime insomnia symptoms and perceived health in the America Insomnia Survey (AIS). Sleep. 2011;34(8):997-1011.

4. Kim JH, Park EC, Yoo KB, Park S. The association between short or long sleep times and quality of life (QOL): results of the Korea National Health and Nutrition Examination Survey (KNHANES IV-V). J Clin Sleep Med. 2015;11(6):625-634.

5. Youngstedt SD, Kripke DF. Long sleep and mortality: rationale for sleep restriction. Sleep Med Rev. 2004;8(3):159-174.

6. Stein MB, Belik SL, Jacobi F, Sareen J. Impairment associated with sleep problems in the community: relationship to physical and mental health comorbidity. Psychosom Med. 2008;70(8):913-919.

7. Taylor DJ, Mallory LJ, Lichstein KL, Durrence HH, Riedel BW, Bush AJ. Comorbidity of chronic insomnia with medical problems. Sleep. 2007;30(2):213-218.

8. Solowij N, Stephens RS, Roffman RA, et al. Cognitive functioning of long-term heavy cannabis users seeking treatment. JAMA. 2002;287(9): 1123-1131.

9. Baglioni C, Battagliese G, Feige B, et al. Insomnia as a predictor of depression: a meta-analytic evaluation of longitudinal epidemiological studies. J Affect Disord. 2011;135(1-3):10-19.

10. Kupfer DJ, Reynolds CF. Management of insomnia. $N$ Engl J Med. 1997;336(5):341-346.

11. Hasler BP, Smith LJ, Cousins JC, Bootzin RR. Circadian rhythms, sleep, and substance abuse. Sleep Med Rev. 2012;16(1):67-81.

12. United Nations Office on Drugs and Crime [webpage on the Internet]. World drug report 2016. United Nations Publication; 2016. Available from: http://www.unodc.org/wdr2016/. Accessed June 2016.

13. Mark TL, Woody GE, Juday T, Kleber HD. The economic costs of heroin addiction in the United States. Drug Alcohol Depend. 2001;61(2): 195-206.

14. Kay DC, Pickworth WB, Neider GL. Morphine-like insomnia from heroin in nondependent human addicts. Br J Clin Pharmacol. 1981;11(2): 159-169.

15. Rosenberg J. Sleep disturbances after non-cardiac surgery. Sleep Med Rev. 2001;5(2):129-137.

16. Shaw IR, Lavigne G, Mayer P, Choinière M. Acute intravenous administration of morphine perturbs sleep architecture in healthy pain-free young adults: a preliminary study. Sleep. 2005;28(6):677-682.

17. Mattick RP, Breen C, Kimber J, Davoli M. Methadone maintenance therapy versus no opioid replacement therapy for opioid dependence. Cochrane Database Syst Rev. 2009;3(3):CD002209.

18. Peles E, Schreiber S, Adelson M. Variables associated with perceived sleep disorders in methadone maintenance treatment (MMT) patients. Drug Alcohol Depend. 2006;82(2):103-110.

19. Dunn KE, Finan PH, Andrew Tompkins D, Strain EC. Frequency and correlates of sleep disturbance in methadone and buprenorphinemaintained patients. Addict Behav. 2018;76:8-14.

20. Teichtahl H, Prodromidis A, Miller B, Cherry G, Kronborg I. Sleepdisordered breathing in stable methadone programme patients: a pilot study. Addiction. 2001;96(3):395-403.

21. Wang D, Teichtahl H, Drummer O, et al. Central sleep apnea in stable methadone maintenance treatment patients. Chest. 2005;128(3):1348-1356.

22. Nguyen CD, Kim JW, Grunstein RR, Thamrin C, Wang D. Respiratory variability during sleep in methadone maintenance treatment patients. J Clin Sleep Med. 2016;12(4):607-616.

23. Nelson AM, Battersby AS, Baghdoyan HA, Lydic R. Opioid-induced decreases in rat brain adenosine levels are reversed by inhibiting adenosine deaminase. Anesthesiology. 2009;111(6):1327-1333.

24. Trksak GH, Jensen JE, Plante DT, et al. Effects of sleep deprivation on sleep homeostasis and restoration during methadone-maintenance: a [31]P MRS brain imaging study. Drug Alcohol Depend. 2010;106(2-3): 79-91.

25. Wang SC, Tsou HH, Chen CH, et al. Genetic polymorphisms in the opioid receptor mu1 gene are associated with changes in libido and insomnia in methadone maintenance patients. Eur Neuropsychopharmacol. 2012;22(10):695-703. 
26. Lydic R, Baghdoyan HA. Sleep, anesthesiology, and the neurobiology of arousal state control. Anesthesiology. 2005;103(6):1268-1295.

27. Watson CJ, Lydic R, Baghdoyan HA. Sleep and GABA levels in the oral part of rat pontine reticular formation are decreased by local and systemic administration of morphine. Neuroscience. 2007;144(1):375-386.

28. Peles E, Schreiber S, Naumovsky Y, Adelson M. Depression in methadone maintenance treatment patients: rate and risk factors. $J$ Affect Disord. 2007;99(1-3):213-220.

29. Angarita GA, Emadi N, Hodges S, Morgan PT. Sleep abnormalities associated with alcohol, cannabis, cocaine, and opiate use: a comprehensive review. Addict Sci Clin Pract. 2016;11(1):9.

30. Burke CK, Peirce JM, Kidorf MS, et al. Sleep problems reported by patients entering opioid agonist treatment. $J$ Subst Abuse Treat. 2008; 35(3):328-333.

31. Chen YZ, Huang WL, Shan JC, Lin YH, Chang HC, Chang LR. Self-reported psychopathology and health-related quality of life in heroin users treated with methadone. Neuropsychiatr Dis Treat. 2013;9:41-48.

32. Magnée EH, de Weert-van Oene GH, Wijdeveld TA, Coenen AM, de Jong CA. Sleep disturbances are associated with reduced health-related quality of life in patients with substance use disorders. Am J Addict. 2015;24(6):515-522.

33. Beswick T, Best D, Rees S, Bearn J, Gossop M, Strang J. Major disruptions of sleep during treatment of the opiate withdrawal syndrome: differences between methadone and lofexidine detoxification treatments. Addict Biol. 2003;8(1):49-57.

34. Wang D, Teichtahl H, Goodman C, Drummer O, Grunstein RR, Kronborg I. Subjective daytime sleepiness and daytime function in patients on stable methadone maintenance treatment: possible mechanisms. J Clin Sleep Med. 2008;4(6):557-562.

35. Stein MD, Herman DS, Bishop S, et al. Sleep disturbances among methadone maintained patients. J Subst Abuse Treat. 2004;26(3):175-180.

36. Kheradmand A, Amini Ranjbar Z, Zeynali Z, Sabahy AR, Nakhaee N. Sleep quality and sexual function in patients under methadone maintenance treatment. Int J High Risk Behav Addict. 2015;4(4):e23550.

37. Peles E, Schreiber S, Domany Y, Sason A, Tene O, Adelson M. Achievement of take-home dose privileges is associated with better-perceived sleep and with cognitive status among methadone maintenance treatment patients. World J Biol Psychiatry. 2014;15(8):620-628.

38. Chiang SC, Chan HY, Chang YY, Sun HJ, Chen WJ, Chen CK. Psychiatric comorbidity and gender difference among treatment-seeking heroin abusers in Taiwan. Psychiatry Clin Neurosci. 2007;61(1):105-111.

39. Mehtry V, Nizamie SH, Parvez N, Pradhan N. Sleep profile in opioid dependence: a polysomnographic case-control study.JClin Neurophysiol. 2014;31(6):517-522.

40. Rose AR, Catcheside PG, McEvoy RD, et al. Sleep disordered breathing and chronic respiratory failure in patients with chronic pain on long term opioid therapy. J Clin Sleep Med. 2014;10(8):847-852.
41. Walker JM, Farney RJ, Rhondeau SM, et al. Chronic opioid use is a risk factor for the development of central sleep apnea and ataxic breathing. J Clin Sleep Med. 2007;3(5):455-461.

42. Sharkey KM, Kurth ME, Anderson BJ, Corso RP, Millman RP, Stein MD. Obstructive sleep apnea is more common than central sleep apnea in methadone maintenance patients with subjective sleep complaints. Drug Alcohol Depend. 2010;108(1-2):77-83.

43. Larance B, Gisev N, Cama E, et al. Predictors of transitions across stages of heroin use and dependence prior to treatment-seeking among people in treatment for opioid dependence. Drug Alcohol Depend. 2018;191: $145-151$.

44. Chen VC, Lin TY, Lee CT, et al. Suicide attempts prior to starting methadone maintenance treatment in Taiwan. Drug Alcohol Depend. 2010;109(1-3):139-143.

45. Lin HC, Chang YP, Wang PW, et al. Gender differences in heroin users receiving methadone maintenance therapy in Taiwan. J Addict Dis. 2013;32(2):140-149.

46. Cheatle MD, Webster LR. Opioid therapy and sleep disorders: risks and mitigation strategies. Pain Med. 2015;16(Suppl 1):S22-S26.

47. Correa D, Farney RJ, Chung F, Prasad A, Lam D, Wong J. Chronic opioid use and central sleep apnea: a review of the prevalence, mechanisms, and perioperative considerations. Anesth Analg. 2015;120(6): $1273-1285$

48. Hoang TV, Ha TT, Hoang TM, et al. Impact of a methadone maintenance therapy pilot in Vietnam and its role in a scaled-up response. Harm Reduct J. 2015;12:39.

49. Bellatorre A, Choi K, Lewin D, Haynie D, Simons-Morton B. Relationships between smoking and sleep problems in black and white adolescents. Sleep. 2017;40(1).

50. Branstetter SA, Horton WJ, Mercincavage M, Buxton OM. Severity of nicotine addiction and disruptions in sleep mediated by early awakenings. Nicotine Tob Res. 2016;18(12):2252-2259.

51. Nordmann S, Lions C, Vilotitch A, et al. A prospective, longitudinal study of sleep disturbance and comorbidity in opiate dependence (the ANRS Methaville study). Psychopharmacology. 2016;233(7); $1203-1213$

52. Lister JJ, Greenwald MK, Ledgerwood DM. Baseline risk factors for drug use among African-American patients during first-month induction/ stabilization on methadone. J Subst Abuse Treat. 2017;78:15-21.

53. Peles E, Linzy S, Kreek M, Adelson M. One-year and cumulative retention as predictors of success in methadone maintenance treatment: a comparison of two clinics in the United States and Israel. J Addict Dis. 2008;27(4):11-25.

54. Saxon AJ, Wells EA, Fleming C, Jackson TR, Calsyn DA. Pre-treatment characteristics, program philosophy and level of ancillary services as predictors of methadone maintenance treatment outcome. Addiction 1996;91(8):1197-1210.
Neuropsychiatric Disease and Treatment

\section{Publish your work in this journal}

Neuropsychiatric Disease and Treatment is an international, peerreviewed journal of clinical therapeutics and pharmacology focusing on concise rapid reporting of clinical or pre-clinical studies on a range of neuropsychiatric and neurological disorders. This journa is indexed on PubMed Central, the 'PsycINFO' database and CAS,

\section{Dovepress}

and is the official journal of The International Neuropsychiatric Association (INA). The manuscript management system is completely online and includes a very quick and fair peer-review system, which is all easy to use. Visit http://www.dovepress.com/testimonials.php to read real quotes from published authors. 